$\begin{array}{ll}\text { Research Square } & \begin{array}{l}\text { Preprints are preliminary reports that have not undergone peer review. } \\ \text { They should not be considered conclusive, used to inform clinical practice, } \\ \text { or referenced by the media as validated information. }\end{array}\end{array}$

\title{
Peripheral nerve activity potential difference among TaiYang, TaiYin, ShaoYang and ShaoYin
}

\author{
Li-yan JIN \\ North Station Hospital of Shanghai \\ Jin-long JIANG \\ Yanbian University Medical College \\ Dao-xuan ZHENG \\ Yanbian University Medical College \\ Chunyu JIN (D2455597388@qq.com ) \\ Yanbian University Medicine College
}

Research

Keywords: Sasang typology, CMAP, F-Wave, Healthy people, PNAP

Posted Date: August 24th, 2020

DOI: https://doi.org/10.21203/rs.3.rs-57433/v1

License: (c) (i) This work is licensed under a Creative Commons Attribution 4.0 International License. Read Full License 


\section{Abstract \\ Objects}

Explain the mechanism of PNAP Characteristic changes for each Korean Medicine Sasang typology.

\section{Methods}

By ways of question form and expert's deliberation, 1000 healthy volunteers are distinguished into corresponding Sasang types, then detect median, ulnar, peroneal, tibial nerves' CMAP and F-Wave, to analyze nerve conduction's characteristic changes between groups or within group.

\section{Results}

$\triangle$ Within group, TaiYin: Median nerve's right side MCV is declined than left side; Ulnar and Peroneal and tibial nerve's left side MCV is declined than right side. ShaoYang: Ulnar and tibial nerve's left side MCV is declined than right side. $(P<0.05)$. Between groups, TaiYin \& ShaoYang: About median and tibial nerve's Distal Amlitude ( DA ), MCV, TaiYin is declined than that of ShaoYang; TaiYin \& ShaoYin: About tibial nerve's DA, MCV, The shortest latency of F-wave ( FL ), TaiYin is declined or extended than that of ShaoYin; ShaoYang \& ShaoYin: For median nerve's MCV, FL, ShaoYin is declined or extended than that of ShaoYang $(P<0.05)$. $\otimes$ Nerve amount rate of FL $\geq 2$ SD ( ShaoYang as standard), for peroneal and tibial nerves, TaiYin is greater than ShaoYang and ShaoYin $(P<0.01)$. $\otimes$ Nerve amount rate which conduction is under-activity, for tibial nerve, TaiYin is greater than ShaoYin ( right ) and ShaoYang ( left ) $(P<0.01)$. $\square$ AMP. / DUR. rate of median nerve: ShaoYin $>$ ShaoYang $>$ TaiYin $(P<0.05)$.

\section{Conclusions}

Median nerve's left side has advantage than right side, however the ulnar and tibial nerve's right side is well than left side, this characteristic is much more obvious in TaiYin; PNAP of ShaoYang is well than TaiYin and ShaoYin; AMP. / DUR. rate of median nerve shows sensitively about constitutional differences.

\section{Significance}

Provide foundation of clinical diagnosis and treatment on a constitutional basis.

\section{Introduction}

The different constitutions will result in the differences of the susceptibility to some pathogenic factors and the tendency of disease development while the course of disease, hence distinguishing constitution is important to predict the occurrence and evolution of disease.

Based on the theory of Tai-Shao-Yin-Yang and five constitutional states of human in $\square$ Ling-shu-Tong-tian区, Jima LI (1837-1891, Korean Hamhung) spit out the Yin-Yang balanced, then divided others into four different constitutional types (Sasang), namely "That the people with large lungs and small liver is called TaiYang; the people with large Liver and small lung is called TaiYin; the people with large splenic and small kidney is called ShaoYang ; the people with large kidney and small spleen is called ShaoYin (Ji-ma LI,1921)."

Basic studies on Sasang typology have been reported successively, such as specific studies on motor nerve and sensory nerve based on Sasang constitutional theory of Korean medicine(Chun-yu JIN, et al., 2012; Chun-yu JIN and Masayuki BABA, 2012), and studies on correlation between acupuncture induction and SEP (He LIU, et al., 2016). However, until now, no research has been found on the characteristic change mechanism of peripheral nerves based on Sasang typology. Therefore, this study mainly explored the specific variation rules and occurrence mechanism of peripheral nerves among Sasang typologies, laying a neurological foundation upon the development of Korean constitutional medicine.

\section{Material And Methods}

\section{General Material}

In the laboratory at room temperature of about 26 degrees, 1,000 healthy volunteers (the Han nationality, because in the previous study we found that the Han nationality has the lowest potential injury rate of terminal nerve (Zhen-he HAN,et al., 2010; Chun-yu JIN and Masayuki BABA, 2009). were distinguished into respective Sasang typology and tested for nerve conduction. Before the experiment, they were informed of the experiment content and signed the informed consent. 1,000 volunteers (427 male), including 34 left-handed; average age of 21.5 years old.

Inclusion criteria: healthy volunteers aged 18 to 34 .

Exclusion criteria: sensory disturbance of limbs; history of peripheral neuropathy. 
2.1 Volunteers filled the questioning table. The questioning table was made mainly based on the diagnosis points from Li Jima's $₫ \mathrm{D}$ ong-Yi-Shou-Shi-BaoYuanland combined the related theories from famous Korean medical books.

2.2 After having research of questioning table, 3-5 clinical experienced Korean medical experts diagnosed each volunteer's Sasang typology by directly observing their appearance, skeletal muscle and pulse.

\section{Nerve conduction examination}

\subsection{Test method}

Test the median, ulnar, peroneal, tibial nerves' CMAP and F-Wave by using Portabook electromyogram inducer(NICOLET Co., USA). All test have been conducted in same time and same condition. During the examination, the subjects took a lying position and were completely relaxed, then stimulated the nerves with saddle stimulator and recorded action potential with surface electrode. The recording electrodes were placed on the abdominals of abductor pollicis brevis, abductor digiti minimi, abductor pedis and extensor digiti brevis respectively, and the standard electrodes were placed on the distal tendons of the same muscle. The stimulating electrodes were located at the wrist, elbow, ankle and knee respectively. The grounding electrode was located between the stimulating electrode and the recording electrode. The distance between the recording electrode and the stimulating electrode was the same on the left and right sides. During the detection, the stimulation intensity gradually increased and stops at $120 \%$ of the CMAP amplitude when it no longer increased. This stimulation intensity was called super stimulation. Using super stimulation to detect F-wave at wrist or ankle, continuously stimulated for 24 times, and recorded the shortest latency of F-wave. The filter amplifier has a bandwidth of $20-2000 \mathrm{~Hz}$, a scanning speed of $5-20 \mathrm{~ms} / \mathrm{cm}$ and a sensitivity of 0.2 $\mathrm{mV} / \mathrm{div}$. The stimulation frequency is $1 \mathrm{~Hz}$ and the time limit was $0.2 \mathrm{~ms}$.

3.2 MCV calculation method and statistical process

Calculation formula: MCV = D / (proximal latency - Total latency). D(Distance) is Body surface distance of ganglion segment measured. Distal Latency (DL) is latency period for wrist or ankle stimulation. Proximal Latency is latency period for stimulation of the elbow or knee. Using SPSS19.0 statistical software to process the measured data. Measurement data, for One-Way ANOVA or independent $t$ - test processing. Counting data for chi-square test. The experiment and analysis process are shown in Figure 1.

(Insert Figure 1)

\section{Results}

\section{Sasang typology data analysis}

\subsection{General situation}

There were 10 volunteers with incomplete test data, which will be eliminated. Among the other 990, 539 were TaiYin, 12 were TaiYang, 269 were ShaoYin and 170 were ShaoYang. See Table 1 for age, weight and length. The age of TaiYin was older than that of ShaoYang $(P<0.01)$. The weight of TaiYin was higher than that of ShaoYang and ShaoYin $(P<0.01)$. The height of TaiYin was higher than that of ShaoYang and ShaoYin $(P<0.01)$.

(Insert Table 1)

\subsection{Sex ratio}

The ratio of men to women among the Sasang groups is shown in Figure 2. There was no significant difference in the ratio of men and women between groups $(P>0.05)$.

(Insert Figure 2)

\subsection{ABO blood type ratio}

See Figure 3. for the $A B O$ blood type ratio among the Sasang groups. In ShaoYin group, the proportion of men and women of type 0 is larger than that of type $A, B$ and $A B$, which is statistically significant $(P<0.05)$.

(Insert Figure 3)

\section{Nerve conduction parameters}

2.1 See Table 2 for the comparison of CMAP and F-wave parameters within and between Sasang groups.

2.1.1 Comparing right and left side within group

\TaiYang: Median nerve's left side FL (F-wave's shortest latency) is extended than right side $(P<0.05)$; Ulnar nerve's right side DL is extended than left side $(P<0.05)$. $\nabla$ TaiYin: Median nerve's right side DL is extended $(P<0.01)$ and MCV is declined $(P<0.01)$; Ulnar nerve's left side DA (Distal of 
Amplitude peak to peak) and MCV is declined ( respectively $P<0.05, P<0.01$ ); Peroneal nerve's right side DL is extended $(P<0.01)$ and DA, MCV is raised ( respectively $P<0.05, P<0.01$ ); Tibial nerve's right side $\mathrm{DL}$ is extended $(P<0.05)$ and MCV is raised $(P<0.01)$ and left side $\mathrm{FL}$ is extended $(P<$ 0.01 ). $\otimes$ ShaoYang: Median nerve's right side MCV is declined ( $P<0.01$ ); Ulnar nerve's right side DA, MCV is raised ( respectively $P<0.05, P<0.01$ ); Peroneal nerve's right side DL is extended $(P<0.05)$; Tibial nerve's left side MCV is declined $(P<0.05)$ and FL is extended $(P<0.01)$. $\otimes$ ShaoYin: Ulnar nerve's left side DA, MCV is declined ( respectively $P<0.05, P<0.01$ ); Peroneal nerve's left side DL is extended $(P<0.01)$ and MCV is raised $(P<0.01$ ); Tibial nerve's left side MCV is declined $(P<0.01)$ and $\mathrm{FL}$ is extended $(P<0.01)$.

\subsubsection{Comparison between groups}

\ TaiYin \& ShaoYang: For median nerve's DL, DA, MCV, FL (R, Right), TaiYin is extended or declinded ( respectively $P<0.05, P<0.05, P<0.01, P<0.05$ ); For ulnar nerve's DA, FL (L, Left), TaiYin is declined or extended ( respectively $P<0.05, P<0.05$ ); For peroneal nerve's FL, TaiYin is extended ( $P<0.01$ ); For tibial nerve's DA, MCV ( L ), FL, TaiYin is extended or declined ( respectively $P<0.01, P<0.05, P<0.01$ ). $\otimes$ Taiyin \& ShaoYin: For median nerve's DL (R), DA, TaiYin is extended or declined ( respectively $P<0.05, P<0.01$ ); For ulnar nerve's DL (L), DA (L), ShaoYin is extended or declined ( respectively $P<0.05, P<$ 0.05 ), however FL $(\mathrm{R})$ is extended in TaiYin $(P<0.05)$; For peroneal nerve's FL, TaiYin is extended $(P<0.01)$; For tibial nerve's DA, MCV, FL, TaiYin is extended or declined ( respectively $P<0.05, P<0.05, P<0.01$ ). $\otimes$ ShaoYang \& ShaoYin: For median nerve's DA, ShaoYin is raised $(P<0.01)$, however MCV, FL is extended or declined ( respectively $P<0.01, P<0.05$ ); For ulnar nerve's DA, ShaoYin is declined $(P<0.01)$. For others, no significant difference is observed $(P>0.05)$.

(Insert Table 2)

\subsection{Neural ratio of FL $\geq 2 S D$}

Regarding FL=2SD of both sides nerves of ShaoYang as the standard (Because the FL data of ShaoYang is the shortest and there is no obvious injury), the neural ratio of FL $\geq 2 S D$ is shown in Figure 4. As the figure shown, $\forall$ Median nerve: ShaoYang right side's ratio is smaller than ShaoYin ( $P<0.01$ ); TaiYin and ShaoYang left side's ratio is smaller than ShaoYin $(P<0.05)$. $\otimes$ Ulnar nerve: TaiYin left side's ratio is greater than ShaoYin $(P<0.01)$. $\otimes$ Peroneal nerve: TaiYin's ratio is greater than ShaoYang and ShaoYin ( respectively $P<0.05, P<0.01$ ). $\otimes$ Tibial nerve: TaiYin's ratio is greater than ShaoYang and ShaoYin ( respectively $P<0.01, P<0.01$ ).

(Insert Figure 4)

\subsection{Neural ratio of poor conduction function}

Under the condition of $\mathrm{DL} \geq 2 \mathrm{SD} \square \mathrm{FL} \geq 2 \mathrm{SD} \square \mathrm{DA} \leq 2 \mathrm{SD} \square \mathrm{MCV} \leq 2 \mathrm{SD}$, the nerves with more than two conditions are considered as having poor conduction function. See Figure 5. In tibial nerve, TaiYin right side's is greater than ShaoYin $(P<0.01)$; TaiYin left side is greater than ShaoYang $(P<0.05)$. The difference between the left and right sides of each nerve in TaiYin has no statistical significance $(P>0.05)$.

(Insert Figure 5)

3 Ratio of AMP. / DUR.

When stimulating wrist, ankle, the AMP. (Amplitude) / DUR. (duration) ratio of the median, ulnar, peroneal and tibial nerves is shown in Figure 6. In median nerve, ShaoYin is greater than TaiYin and ShaoYang (respectively $P<0.01, P<0.05$ ); ShaoYang is greater than TaiYin ( $P<0.01$ ). In tibial nerve, ShaoYang is greater than TaiYin $(P<0.01)$.

(Insert Figure 6)

\section{Discussion}

The proportion of each Sasang typology is $1 \%$ of TaiYang, $54 \%$ of TaiYin, $17 \%$ of ShaoYang and $27 \%$ of ShaoYin. aDong-Yi-Shou-Shi-Bao-Yuan $\llbracket$ said "At this time, we can see 10,000 people in a county. In a nutshell, there are 5,000 people is TaiYin, 3,000 is ShaoYang, 2,000 is ShaoYin, and only three or four people is TaiYang." Myung Jin Oh, et al. (2013) used QSCCII method to diagnose 40 healthy people, with result of that TaiYin and ShaoYin accounted for $39.0 \%$ and ShaoYang for $19.5 \%$ respectively. The Sasang typology proportion of 265 healthy volunteers reported by Kim is $41.9 \%$ in TaiYin, $37.6 \%$ in ShaoYin and $20.5 \%$ in ShaoYang (Sun Ho Kim, 1996). The above results are basically consistent with this study. The results showed that the weight and height of TaiYin were higher than those of ShaoYang and ShaoYin. The traditional medical books recorded that "TaiYin is well-developed and obese (Zhenan XUAN, 2005)". Chun-yu JIN, et al. (2012) reported that the body mass and height of TaiYin were higher than those of ShaoYang and ShaoYin. The above results are basically consistent with this study. This time, there were more men are ShaoYin and more women are TaiYin, but the difference was not statistically significant. Sun Ho Kim, et al. (1996) reported that in men, the number of ShaoYin is more than that of TaiYin, while in women, the number of TaiYin is more than that of ShaoYin, which is very similar to this result. Within ShaoYin group, There is a significant difference between men and women in type $\mathrm{O}, \mathrm{A}, \mathrm{B}$ and $\mathrm{AB}$, which is statistically significant. The characteristics of ShaoYin's viscera are "large kidney and small spleen", the function of lower energizer (Kidney, baldder, large intestine, small intestine) tends to strong. Its reproductive capacity is stronger than other Sasang typologies. Bi-yun Zhang, et al. (2017) found that the highest proportion of premature ovarian failure (POF) patients was type A, and the lowest was type O. It just shows that the function of kidney is more powerful with type 0 . 
There are few specimens of TaiYang, so we should study them later. The right side of DL and MCV of the median nerve in the TaiYin is extended or declined. The right side of ulnar, peroneal and tibial nerves are conductive advantage. $96 \%$ of the volunteers were right-handed, and they used their right hands to write more in their study and life, besides TaiYin has the characteristics of large liver, small lung, astringent Qi and turbid blood, which leads to more microcirculatory diseases than other Sasang typologies. In the same right hand, the ulnar nerve is conductive to the right side, suggesting that there may be a potential conduction disorder in the carpal tunnel. Ying LIU, et al. (2017) examined 180 CTS patients, found that 78 of the left hand and 102 of the right hand had CTS, which indicated that the incidence rate of right hand was higher than that of left hand. Compared with asymptomatic or healthy people, the SCV of median nerve declined and DL extended in patients with symptomatic CTS, but there was no significant difference in DA reduction (Yao YAO, 2017). It is speculated that the right hand is the most flexible and commonly used limb, and the risk of injury is higher than that of the left hand. 口Huang-Di-Nei-Jing $\square$ said "the sprit is well managed when Yin-Yang is balanced". As Yang has the function of warming and promoting Yin, TaiYin who has more Yin characteristic would cause slow metabolism and endogenesis of phlegm dampness. This would lead to Physiological and pathological environment of terminal nerve. This may be the reason why the conduction function of the right side of the median nerve is lower than that of the left side.

DA and MCV of ulnar nerve and MCV and FL of tibial nerve in TaiYin, ShaoYang and ShaoYin were extended or declined on the right side. That says, the right side of ulnar and tibial nerve's conduction is superior. In the initial state of study of 100 healthy subjects, the right side of ulnar and tibial nerve's MCV was increased than the left (Chun-yu JIN, et al., 2011). The PA of the median nerve of TaiYin is higher on the left side; The PA of the ulnar nerve of ShaoYin is higher on the right side; The MCV of the ulnar and tibial nerves of ShaoYang is increased on the right side. In patients with diabetic peripheral neuropathy (DPN), DL of median, ulnar, posterior tibial and right side of common peroneal nerve extended, DA and MCV is declined (Guo-ping XING, 2009). Through acupuncture and moxibustion, MCV of the left median, ulnar and right peroneal and tibial nerves increased significantly (Dong-cai WANG,2017). It is speculated that the right handedness is one of the possible factors for the conduction advantage of the right limb.

DL of peroneal nerve of TaiYin, ShaoYang and ShaoYin was extended on the right side, and MCV was increased on the right side. Zhen-he HAN, et al. (2010) reported that the DA of peroneal nerve of healthy Korean was lower and DL was longer than that of Chinese Korean.Cai-hong Cai-hong Ma and Xiao-yun HUANG (2017) compared the Hui nationality group with the mixed group and the Han nationality group, the Hui nationality group's DL of common peroneal nerve extended, MCV and DA declined. Anatomically, the position of the peroneal nerve is shallow so it is vulnerable to external influences such as physical squeeze. It is speculated that living habits (such as kneeling, sitting, etc.) are the important reasons for the potential injury of the anterior peroneal nerve of ankle joint.

In this study, DL, FL (R), DA, MCV of median nerve and DA,FL ( $L$ ) of ulnar nerve and DA, MCV, FL ( $L$ ) of peroneal nerve are extended or declined in TaiYin than that of ShaoYang. Chun-yu JIN, et al. (2012) showed that DL and FL of median, ulnar, tibial and peroneal nerves in TaiYin were extended than those in ShaoYang, which was consistent with the results of this study. It is said in₫Dong-Yi-Shou-Shi-Bao-Yuan】that "Tai-Shao-Yin-Yang has different Visceral pattern, which depends on variation of Yin-Yang balance (Zhe-nan XUAN, 2015)." [Ling-Shu-Ni-Shun-Fei-Shou [said "Fat people, whose blood is black and turbid, whose Qi is unsmooth and slow. Thin people, whose blood is clear and Qi is smooth." Above shows that different amount of Yin-Yang in viscera produces four different constitutional typology, namely Sasang. The internal environmental factors of different constitutional typology directly affect the physiological and pathological changes of nerve, and make them change accordingly.

DL (R), DA of median nerve, DA, MCV, FL of tibial nerve, FL of peroneal nerve extended or declined in the TaiYin than that of ShaoYin. DL (L), DA ( $L$ ) of ulnar nerve extended or declined in ShaoYin than that of TaiYin. FL (R) of median nerve is extended in TaiYin than that of ShaoYin. According to traditional Chinese Medicine (TCM) that Yang is functional for warming and motivating. Meanwhile, Yin is functional for moist and mourishing. Since TaiYin has larger Yin/Yang balance than ShaoYin has, so the nerve conduction function of TaiYin tends to weak. Li-yan JIN (2018) examined the conduction function of the median nerve, and found that FL $(\mathrm{L})$ is extended in TaiYin than that of ShaoYang and ShaoYin, and FL (R) in TaiYin was extended than that of ShaoYin $(P<0.05)$. Sheng-ai PIAO (2019) tested the conduction function of peroneal nerve. The results showed that DL and FL in TaiYin were extended than that of ShaoYang and ShaoYin. DL, MCV in TaiYin and ShaoYin were all right side extended than that of left side.

DA of median nerve in ShaoYin was extended than that of ShaoYang, MCV and FL of median nerve in, and DA of ulnar nerve in ShaoYin was extended or declined than that of ShaoYang $(P<0.05)$. ShaoYang has the constitutional characteristic of "a bit more amount of Yang than Yin; Upper excess and lower deficiency". ShaoYin has the constitutional characteristic of "a bit more amount of Yin than Yang; Lower excess and upper deficiency". The median and ulnar nerves of the upper limbs of ShaoYang has nerve conduction advantage than ShaoYin, but there is no significant difference in nerve conduction between the lower limbs. It is speculated that the Qi of ShaoYang is rather stronger, and ShaoYin is rather easier to lose the QiXue, and the spleen tends to weak and cold so the significant difference of PNAP could be observed. According to Gang-hua YAN, et al. (2010) TCM Constitution can be divided into three types: "Qi and Yin deficiency", "phlegm and blood stasis", "Yang deficiency and blood stasis". Their median, ulnar and peroneal nerve conduction parameters are MCV is gradually slowed down, DA is gradually declined, DL is gradually extended. Qi LI (2010) reported that among the various types of Type 2 diabetic peripheral neuropathy, Toronto's clinical scoring system was used to evaluate the degree of nerve injury that "Deficiency of yin and hyperactivity of heat" group has the mildest nerve injury. The significant difference of MCV is between "Yin deficiency and heat excess" group and "blood stasis and venation" group, the former is lighter than the latter. Yuan-bo Xin, et al. (2015) investigated and analyzed the Sasang typology and TCM constitution by questionnaire, found that the TaiYang is closely related to the hygrothermal constitution, the TaiYin is closely related to the phlegm and dampness constitution, the ShaoYang is closely related to the Yin deficiency constitution, and the ShaoYin is closely related to the Yang deficiency constitution. Go Jung-hyun, et al. (2010) observed that phlegm dampness constitution is similar to the TaiYin; Qi deficiency, Yang deficiency and Qi depression are similar to ShaoYin; Yin deficiency constitution is similar to ShaoYang; Damp heat constitution is similar to TaiYang and ShaoYang; Blood stasis constitution is similar to ShaoYin and TaiYin. 
For median nerve, ShaoYin is greater than ShaoYang and TaiYin $(\mathrm{L})(P<0.05)$; For ulnar nerve, TaiYin is greater than ShaoYin $(\mathrm{L})(P<0.01)$; For peroneal and tibial nerve, TaiYin is greater than ShaoYang and ShaoYin $(P<0.01)$. In addition, the ratio of nerves with low conduction function in tibial nerve, that TaiYin was higher than ShaoYin (R) and ShaoYang (L). It is said in DDong-Yi-Shou-Shi-Bao-Yuan $\rrbracket$ that "The Qi of grief and anger rises, the Qi of like and happy drops. If you have too much rising Qi, the lower energizer will be damaged. If you have too much descending Qi, the upper energizer will be damaged. The temperament characteristics of ShaoYin is freely like and deeply happy. The Qi of descent is too much to damage the upper energizer. Their internal organs are characterized by small spleen and large kidney and their body shape is that upper body is thin, while the lower body is thick. ShaoYin have small spleen which functions to transport and disperse essence, so they are easily to lose Qi-Xue and the spleen tends to be weak and cold.

Therefore, the proportion of injured nerves in upper limbs is significantly higher than that in lower limbs. The temperament characteristics of ShaoYang is suddenly grief and deeply angry, so the Qi of rising is too much to damage the lower energizer. Their internal organs are characterized by small kidney and large spleen and their body shape is that lower body is thin, while the upper body is thick. ShaoYin have more amount of Yang with less amount of Yin that they have weak kidney function so the kidney is easy to be overheated. Therefore, their nerve conduction function is rather stronger and the ratio of FL $\geq$ 2SD is the smallest. The temperament characteristics of TaiYin is freely happy and deeply like. Their internal organs are characterized by small lung and large liver and their body shape is characterized by waist hypertrophy. Because of they have small lung and the weakness of promoting blood, TaiYin are prone to be in the state of "blood turbid and Qi astringent. The proportion of injured nerves in lower limbs was significantly higher than that in ShaoYin and ShaoYang.

Tibial nerve hypofunction rate is TaiYin (R) greater than ShaoYin $(P<0.01)$; TaiYin $(\mathrm{L})$ greater than ShaoYang $(P<0.05)$. It can be seen that constitutional specificity leads to the susceptibility of the body to certain diseases. Compared with ShaoYang and ShaoYin, TaiYin are more likely to suffer from potential nerve damage because of their constitution characteristics of "big liver, small lung, turbid blood and astringent Qi"

There is a significant correlation between AMP. and DUR. It is a stable electrical characteristic of skeletal muscle motor unit, when it exceeds a certain time limit, the variability of amplitude becomes larger (Yang QIN, et al., 2011). The ratio of the median nerve is ShaoYin > ShaoYang > TaiYin, i.e. DL is ShaoYang/ShaoYin < TaiYin; Proximal latency is ShaoYang < ShaoYin/TaiYin; Proximal duration is ShaoYang/ShaoYin < TaiYin; DA/PA is ShaoYin > ShaoYang > TaiYin. The integrity of CMAP wave-forms in the median nerve of ShaoYin was verified, suggesting that it can be used to distinguish the Sasang typology. The change of tibial nerve ratio suggests that the conduction function of TaiYin is lower than that of ShaoYin and ShaoYang. It was found in previous studies that among the different TCM Syndromes of CTS patients, the median nerve conduction function of healthy volunteers is better than that of "cold dampness blocking collaterals" group, then the "cold dampness blocking collaterals" group is better than "Qi and Yin deficiency group" (Shuo ZHANG, 2011). Among the different TCM Syndromes of DPN patients, the most significant difference of MCV and DA had been observed between "Yang deficiency, cold coagulation" group and "phlegm and blood stasis" "Qi and yin deficiency" groups, especially in the median nerve (Guo-xin GAO, 2013). In addition, DPN nerve conduction test is characterized by high abnormal rate of MCV in tibial and median nerve (Zhu LIU, et al., 2014). In aerobic exercise group, DL and MCV of common peroneal and posterior tibial nerves were significantly improved (Yan WANG, 2015).

The above median, ulnar, peroneal and tibial nerve conduction parameters show that the nerve conduction function of ShaoYang with overheated constitution is superior to that of TaiYin with astringent Qi constitution and ShaoYin people with supercooled constitution. The ratio of AMP. / DUR. of median nerve can sensitively reflect the difference of constitutions, and can be used in the differential diagnosis of Sasang typology.

\section{Conclusions}

In conclusion, median nerve's left side has an advantage than right side, however the ulnar and tibial nerve's right side is well than left side, this characteristic is much more obvious in TaiYin; PNAP of ShaoYang is well than TaiYin and ShaoYin; AMP. / DUR. rate of median nerve shows sensitively about constitutional differences.

\section{Abbreviations}

\section{PNAP}

Peripheral Nerve Activity Potential

CMAP

Compound Muscle Activity Potential

DL

Distal Latency

DA

Distal Amlitude peak to peak

MCV

Motor Conduction Velocity

FL

The shortest Latency of F-wave

SD

Standard

$\mathrm{R}$

Right 
Somatosensory Evoked Potential

D

Distance

AMP. / DUR.

Amplitude / Duration

POF

Premature Ovarian Failure

CTS

Carpal Tunnel Syndrome

SCV

Sensory Conduction Velocity

DPN

Diabetic Peripheral Neuropathy

TCM

Traditional Chinese Medicine

PA

Proximal Amplitude

\section{Declarations}

\section{Ethics approval and consent to participate}

After deliberation by the medical ethics committee of Yanbian University, the experimental design and implementation plan of the study fully considered the principles of safety and fairness. The research content does not constitute harm and risk to the subjects. The recruitment of the subjects will be based on the principles of voluntary and informed consent, and try to protect the privacy of the subjects as much as possible. There is no conflict of interest in the research content and results.

Medical College of Yanbian University understood the purpose and content of this experiment about the nerve conduction velocity detection in detail, and agreed to assist as the experimenter.

\section{Consent for publication}

Not applicable.

\section{Availability of data and materials}

Please contact author for data requests.

\section{Funding}

This work was supported by the National Natural Science Foundation of China (No. 81760903) and Jilin Science and technology development plan project(No. 20180101304jc).

\section{Competing interests}

The authors declare that there is no conflict of interests regarding the publication of this manuscript.

\section{Authors' contributions}

Li-yan JIN carried out the NCS studies, Jin-long JIANG coordinated all processes, Dao-xuan ZHENG performed statistical analysis. Chun-yu JIN mainly participated in the design and conduction of the study. All authors read and approved the final manuscript.

\section{Acknowledgements}

Not applicable.

\section{References}

1. Ji-ma LI. Original. Rong-jun JIN Edit.ロDong-yi-shou-shi-bao-yuan『[M]. Third Edition. Beijing: Bowen Library, 1921. P7.

2. Chun-yu JIN. Chang-qing LIN, Zhen-yu LIU. Study about motor nerve conduction function of Sasang people in Korean medicine [J]. Lishizhen Medicine Materia Medica Research, 2012, 23 (03): 755-8. 
3. Chun-yu JIN. Masayuki BABA. Study about sensory nerve conduction function of Sasang people with Korean medicine [J]. China Journal of Traditional Chinese Medicine Pharmacy, 2012, 27 (05): 1257-61.

4. He LIU. Chun-yu JIN, Lei WANG. Tian-yue ZHANG. Needling $\mathrm{I}^{1} \mathbf{4}^{2}$ esponse Sensitivity and SEP [J]. Liaoning Journal of Traditional Chinese Medicine, 2016, 43 (06): 1128-31.

5. Zhen-he HAN. Chun-yu JIN, Zhen-yu LIU. Relations between Peripheral Nerve Conduction Parameter and Living Habits of Healthy Subjects [J]. Chinese Journal of Ethnomedicine Ethnopharmacy, 2010, 19 (03): 77-9.

6. Chun-yu JIN. Masayuki BABA. The meaning of A-Wave appearing in limb nerve conduction [J]. Journal of Medical Science Yanbian University, 2009, 32 (03): 178-80.

7. Oh MJ. Youn Shim, Ho Sueb Song. Sasang Constitutional Diagnosis by Portable Ryodoraku Device. The Acupuncture, 2013,30 (4): $161-7$.

8. Sun Ho Kim. A study on the standardization of QSCCII. The Journal of Korean oriental medical society.1996,17 (2):337-393.

9. Zhe-nan XUAN

口Dong-Yi-Shou-Shi-Bao-Yuan $\llbracket$ explanation [M].YanJi

Zhe-nan XUAN.]Dong-Yi-Shou-Shi-Bao-Yuan־explanation [M].YanJi: Yanbian people's Publishing House, 2005. P309.

10. Bi-yun ZHANG. Li-wei HAN,Mei-qing XIE. Meng-zhao WANG, An LIANG. Xiu-mei LI, et al. Study of correlation between premature ovary failure and ABO blood types or Type A behavior pattern. [J]. Guangdong Medical Journal, 2017, 38 (03): 383-6.

11. Ying LIU. Yan GUO, Wenping JIANG. Hong LEl. The Diagnostic Value of Carpal Tunnel Syndrome by Ultrasonography [J]. Chinese Journal of Ultrasound in Medicine, 2017, 33 (06): 537-9.

12. Yao YAO. The application of nerve conduction test and ultrasonography in patients with carpal tunnel syndrome[D]. Wannan Medical College, 2017.

13. Chun-yu JIN. Rui WANG, Mashuki BABA. Comparison between right and left motor nerves conduction parameters of healthy adults [J]. Progress in Modern Biomedicine, 2011, (5): 815-818.

14. Guo-ping XING. Comparative Study of Neuroeletrophysiological Methods on Evaluating the Function of Peripheral Nerves Diabetic Patients [D].Tianjin Medical University, 2009.

15. Dong-cai WANG. Effect of Acupuncture-moxibustion in Assisting the Treatment of Diabetic Peripheral Neuropathies on the Recovery and Prognosis of Neurological Function [J]. Shanghai Journal of Acupuncture and Moxibustion, 2017 (12): 1439-1442.

16. Cai-hong MA. Xiao-yun HUANG. Study on the differences of nerve conduction of lower limbs in general population with different nationalities in Xining region [J]. Journal of Clinical Neurology. 2017 (02).

17. Li-yan JIN. Study on the correlation between acupoint temperature and PNCS bansed on the theory of sasang constitutional medicine [D]. Yanbian University, 2018.

18. Sheng-ai PIAO. The relationship between the CCT characteristics of the four-image constitution and the potential damage or peripheral nerve [D]. Yanbian University, 2019.

19. Gang-hua YAN. Relationship between Types of Tradition Chinese Medicine syndrome of Diabetic Peripheral Neuropathy and Neurophysiological Feature [D]. Fujian University f Traditional Chinese Mediclne, 2010.

20. Qi LI. Type2 Diabetes around neuropathy the dialectical divided and laboratory tests of the relevance of clinical research [D]. Hubei University $f$ Traditional Chinese Mediclne, 2010.

21. Yuan-bo XIN. Wei-jian JIN, Guo-quan WEN. Yu-jin XU, et al. The study of Relationship between Korean Sasang constitution and traditional Chinese Medicine Constitution Type [J]. Journal of Medicine Pharmacy of Chinese Minorities, 2015, 21 (05): 71-2.

22. Go Jung-hyun. Gwon Hui-wan, Sung Sun-hui. The Comparative Study Constitutions of Traditional Chinese Medicine and Sasang Constitutional Medicine. Intentional Journal of Integrative Alternative Medicine. 2010;6((1):):57-67.

23. Yang QIN. Chao-hui CHEN,Li LING. Correlation between MUPs duration and amplitude in limb muscle [J]. Journal of Chongqing Medical University, 2011, 36 (01): 58-60.

24. Shuo ZHANG. Relationship between TCMS yndrome of CarPal Tunnel Syndrome and Neurophysiological Features [D]. Fujian University f Traditional Chinese Mediclne, 2011.

25. Guo-xin GAO. Study on relationship between syndrome of traditional Chinese medicine and electromyography examination in diabetic peripheral neuropathy [D]. Sandong University $f$ Traditional Chinese Mediclne, 2013.

26. Zhu LIU. Zhi-rong JIA, Ting-ting WANG. Xin SHI, Wei LIANG. Characteristics of nerve conduction in type 2diabetic peripheral neuropathy [J]. Chinese Journal of Diabetes, 2014, 22 (12): 1060-3.

27. Yan WANG. Effect of different modes of exercise on nerve conduction in patients with diabetic peripheral neuropathy [D]. Tianjin Medical Univerity, 2015.

\section{Tables}

Table 1 General data of TaiYang, TaiYin, ShaoYang ShaoYin (Mean \pm SD) 


\begin{tabular}{|llll|}
\hline Sasang & Age & weight $\varangle \mathrm{kg} \triangle$ & height $\backslash \mathrm{cm} \rrbracket$ \\
\hline TaiYang & $22.2 \pm 1.5$ & $55.6 \pm 7.0$ & $166.9 \pm 6.1$ \\
\hline TaiYin & $21.6 \pm 1.8$ & $59.9 \pm 11.3$ & $166.8 \pm 8.2$ \\
\hline ShaoYang & $21.2 \pm 1.6 \triangle \triangle$ & $52.8 \pm 10.9 \triangle \triangle$ & $164.6 \pm 7.5 \triangle \triangle$ \\
\hline ShaoYin & $21.4 \pm 1.8$ & $52.6 \pm 9.7 \triangle \triangle$ & $164.8 \pm 7.8 \triangle \triangle$ \\
\hline
\end{tabular}

Note: $\triangle \triangle P<0.01 \rrbracket \triangle$ Compared with TaiYin®One-way Anoval

Table 2 CMAP and F-wave parameters of the right and left side

\begin{tabular}{|c|c|c|c|c|c|c|c|c|}
\hline & \multicolumn{2}{|l|}{ TaiYang } & \multicolumn{2}{|l|}{ TaiYin } & \multicolumn{2}{|l|}{ ShaoYang } & \multicolumn{2}{|l|}{ ShaoYin } \\
\hline & Right & Left & Right & Left & Right & Left & Right & Left \\
\hline \multicolumn{9}{|c|}{ Median } \\
\hline $\mathrm{DL}$ & $3.16 \pm 0.40$ & $3.11 \pm 0.29$ & $3.19 \pm 0.39 * \star$ & $3.13 \pm 0.37$ & $3.06 \pm 0.38 \triangle \triangle$ & $3.04 \pm 0.35 \triangle$ & $3.12 \pm 0.44 \triangle$ & $3.11 \pm 0.45$ \\
\hline DA & $11.45 \pm 3.94$ & $12.01 \pm 2.06$ & $10.51 \pm 3.54$ & $10.30 \pm 3.36$ & $11.21 \pm 3.47 \triangle$ & $11.24 \pm 3.00 \triangle \Delta$ & $12.24 \pm 3.81 \Delta \mathbf{\Delta} \Delta \Delta$ & $12.45 \pm 3.57 \Delta \Delta \Delta \Delta$ \\
\hline MCV & $60.33 \pm 2.50$ & $60.17 \pm 3.76$ & $60.52 \pm 4.15^{\star \star}$ & $61.15 \pm 4.57$ & $61.75 \pm 3.95^{\star \star \Delta} \Delta \Delta$ & $62.64 \pm 3.93 \triangle \Delta$ & $60.58 \pm 3.85 \Delta \Lambda$ & $60.86 \pm 4.18 \Delta \Lambda$ \\
\hline $\mathrm{FL}$ & $23.68 \pm 1.27 *$ & $24.1 \pm 1.15$ & $23.84 \pm 1.71$ & $23.81 \pm 1.67$ & $23.53 \pm 1.66 \triangle$ & $23.62 \pm 1.62$ & $24.10 \pm 1.87 \Delta \Delta$ & $24.04 \pm 1.90^{\Delta}$ \\
\hline \multicolumn{9}{|l|}{ Ulnar } \\
\hline $\mathrm{DL}$ & $2.48 \pm 0.30 *$ & $2.41 \pm 0.38$ & $2.48 \pm 0.30$ & $2.46 \pm 0.29$ & $2.46 \pm 0.32$ & $2.46 \pm 0.32$ & $2.50 \pm 0.32$ & $2.52 \pm 0.32^{\triangle}$ \\
\hline DA & $10.11 \pm 2.30$ & $10.83 \pm 2.58$ & $10.61 \pm 2.55^{\star}$ & $10.43 \pm 2.57$ & $11.28 \pm 2.62^{\star \Delta \Delta}$ & $10.92 \pm 2.73^{\triangle}$ & $10.44 \pm 3.07 \star \Delta \Delta$ & $10.04 \pm 2.59 \triangle \boldsymbol{\Delta} \Delta$ \\
\hline MCV & $60.36 \pm 4.34$ & $59.42 \pm 4.03$ & $59.43 \pm 5.70 * \star$ & $57.99 \pm 5.99$ & $59.77 \pm 5.48^{\star \star}$ & $58.74 \pm 6.19$ & $59.31 \pm 5.85^{\star \star}$ & $57.92 \pm 5.88$ \\
\hline $\mathrm{FL}$ & $24.25 \pm 1.48$ & $24.43 \pm 1.60$ & $24.48 \pm 1.90$ & $24.46 \pm 1.91$ & $24.18 \pm 1.86$ & $24.09 \pm 1.82 \triangle$ & $24.17 \pm 1.86^{\triangle}$ & $24.24 \pm 1.78$ \\
\hline \multicolumn{9}{|c|}{ Peroneal } \\
\hline DL & $4.00 \pm 0.60$ & $4.00 \pm 0.67$ & $3.96 \pm 0.59 \star \star$ & $3.88 \pm 0.59$ & $3.97 \pm 0.64^{\star}$ & $3.90 \pm 0.58$ & $3.98 \pm 0.61$ ** & $3.86 \pm 0.52$ \\
\hline DA & $7.46 \pm 4.21$ & $6.82 \pm 2.88$ & $7.83 \pm 3.33^{*}$ & $7.52 \pm 3.40$ & $8.06 \pm 3.68$ & $7.78 \pm 3.71$ & $7.54 \pm 3.53$ & $7.77 \pm 3.44$ \\
\hline MCV & $53.75 \pm 2.01$ & $51.42 \pm 4.38$ & $52.22 \pm 3.77 * \star$ & $51.64 \pm 3.64$ & $52.02 \pm 3.45$ & $51.73 \pm 3.74$ & $52.40 \pm 3.88^{\star \star}$ & $51.64 \pm 3.78$ \\
\hline $\mathrm{FL}$ & $43.41 \pm 2.58$ & $43.92 \pm 3.70$ & $44.10 \pm 3.59$ & $44.19 \pm 3.62$ & $43.14 \pm 3.09 \triangle \triangle$ & $43.27 \pm 3.13 \triangle \Delta$ & $43.22 \pm 3.08 \triangle \Delta$ & $43.09 \pm 2.95 \triangle \triangle$ \\
\hline \multicolumn{9}{|l|}{ Tibial } \\
\hline $\mathrm{DL}$ & $3.93 \pm 0.47$ & $3.71 \pm 0.56$ & $3.94 \pm 0.66^{\star}$ & $3.88 \pm 0.67$ & $3.93 \pm 0.70$ & $3.88 \pm 0.68$ & $3.92 \pm 0.70$ & $3.90 \pm 0.63$ \\
\hline DA & $16.88 \pm 8.58$ & $16.88 \pm 7.85$ & $15.80 \pm 7.25$ & $16.00 \pm 7.30$ & $18.47 \pm 7.61 \triangle \triangle$ & $17.97 \pm 7.77 \triangle \triangle$ & $17.27 \pm 7.76 \triangle \triangle$ & $17.13 \pm 7.50^{\triangle}$ \\
\hline MCV & $49.17 \pm 3.88$ & $49.25 \pm 4.97$ & $47.82 \pm 4.95^{\star \star}$ & $46.45 \pm 5.13$ & $48.19 \pm 4.77^{\star}$ & $47.45 \pm 5.23^{\triangle}$ & $48.58 \pm 5.01 \star \star \triangle$ & $47.25 \pm 4.97 \triangle$ \\
\hline $\mathrm{FL}$ & $43.53 \pm 2.65$ & $43.44 \pm 3.36$ & $43.96 \pm 3.51^{\star \star}$ & $44.15 \pm 3.50$ & $42.91 \pm 2.87 * \star \Delta \Delta$ & $43.21 \pm 2.71 \triangle \Delta$ & $42.97 \pm 3.00 * \star \Delta \triangle$ & $43.29 \pm 3.22 \triangle \triangle$ \\
\hline
\end{tabular}

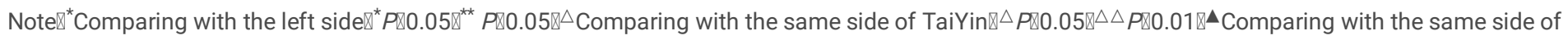

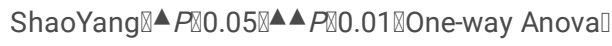

\section{Figures}




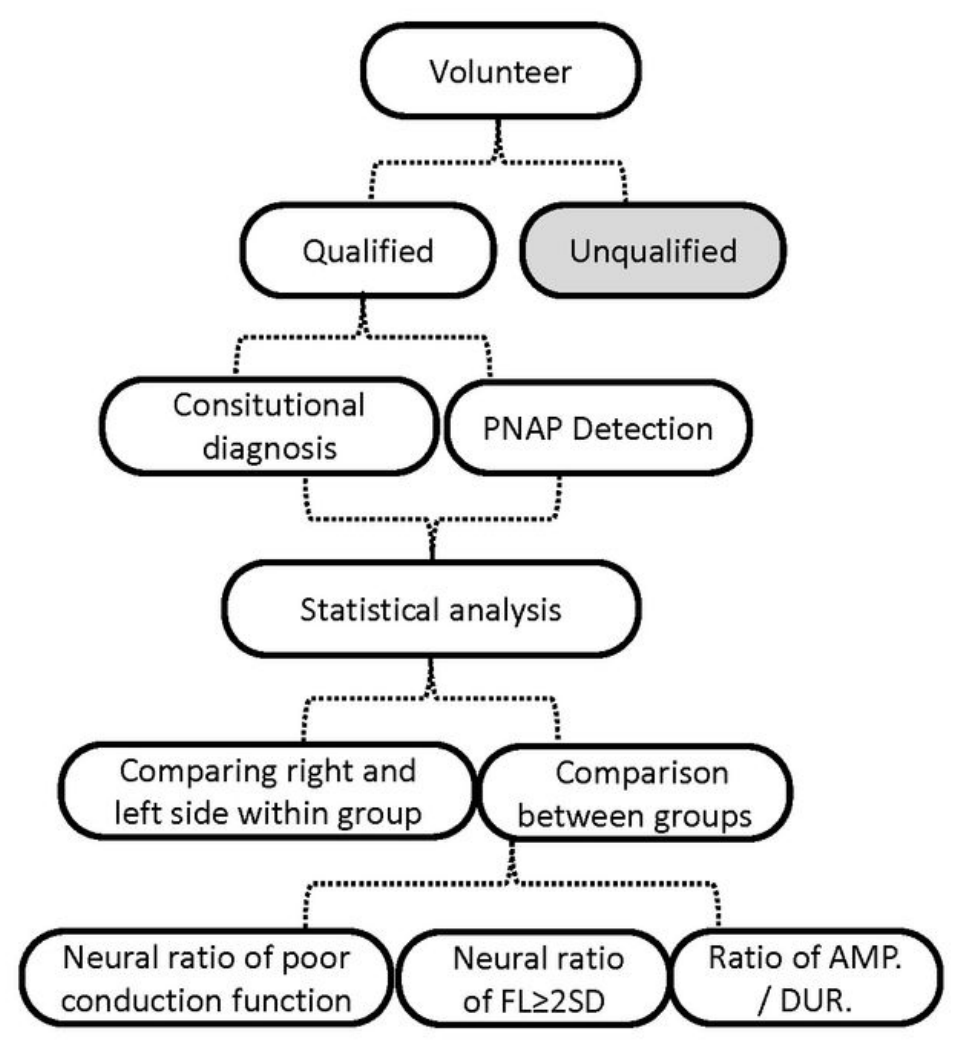

Figure 1

Experiment and analysis process

Page 10/15 


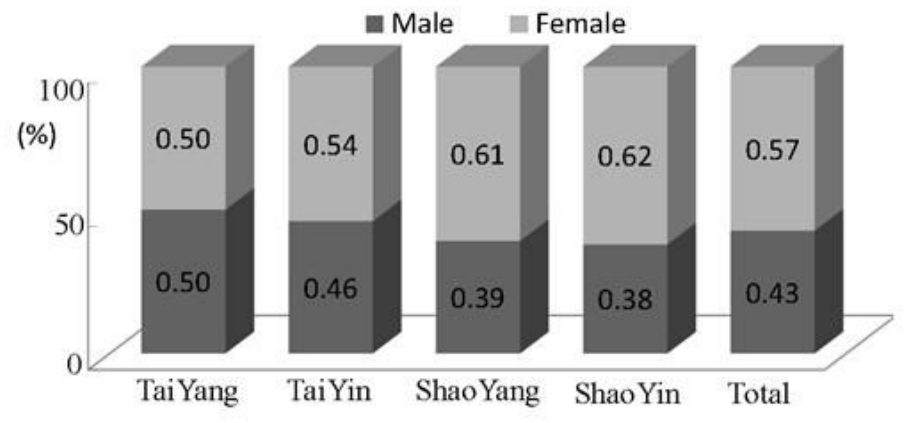

Figure 2

Rate of men and women in Sasang groups 


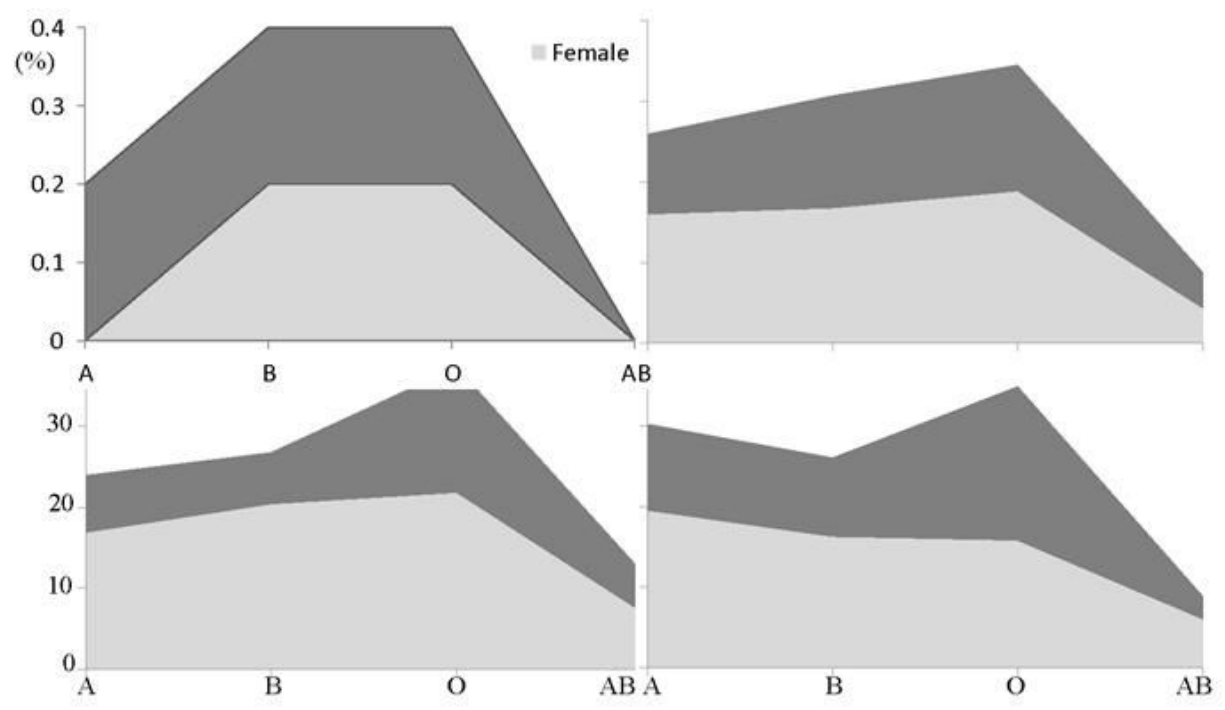

Note: Upper left is TaiYang; Upper right is TaiYin; Bottom left is Shao Yang; Bottom right is ShaoYin. Chi-square Test.

\section{Figure 3}

ABO blood type rate in Sasang groups (unit: \%) Note囚Upper left is TaiYang; Upper right is TaiYin; Bottom left is ShaoYang; Bottom right is ShaoYin. Chisquare Test. 


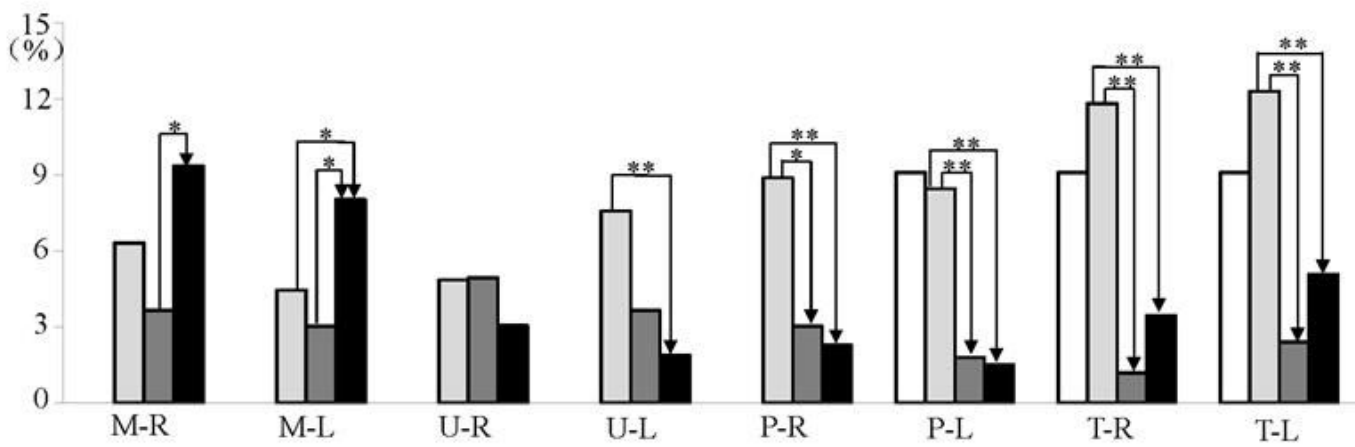

Note:Neural ratio formula $=$ Nerves count for FL $\geq 2 \mathrm{SD} /$ Nerves count for $\mathrm{FL}<2 \mathrm{SD} \%$,

White: Tai Yang; Light grey: TaiYin; Dark grey: Shao Yang; Black: Shao Yin, (M, Median; U, Ulnar; P,

Peroneal; T, Tibial; R, Right; L, Left). Same as below.

Figure 4

Neural ratio of FL $\geq 2 S D$ in Median, Ulnar, Peroneal, Tibial nerves Note:Neural ratio formula = Nerves count for FL $\geq 2 S D$ / Nerves count for FL $邓 2 S D \% \bigotimes$ White: TaiYang; Light grey: TaiYin; Dark grey: ShaoYang; Black: ShaoYin, (M, Median; U, Ulnar; P, Peroneal; T, Tibial; R, Right; L, Left). Same as below. 


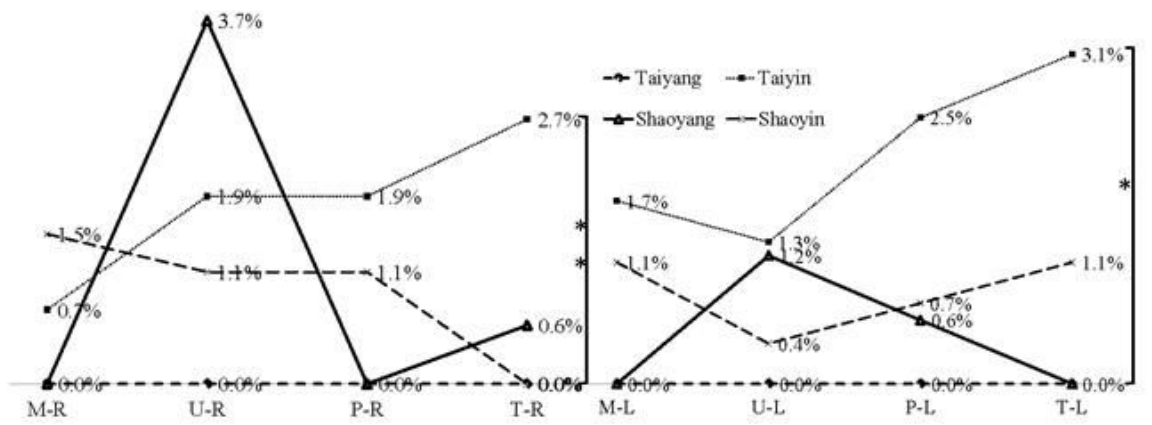

Note: Ratio of people with more than 2 conditions among the conditions DI $\geq 2 \mathrm{SD}$,

$\mathrm{FL} \geq 2 \mathrm{SD}, \mathrm{DA} \leq 2 \mathrm{SD}, \mathrm{MCV} \leq 2 \mathrm{SD}$.

\section{Figure 5}

Neural ratio of any two or more parameters beyond the 2SD range (unit: \%) Note: Ratio of people with more than 2 conditions among the conditions $D L \geq 2 S D, F L \geq 2 S D, D A \leq 2 S D, M C V \leq 2 S D$. 


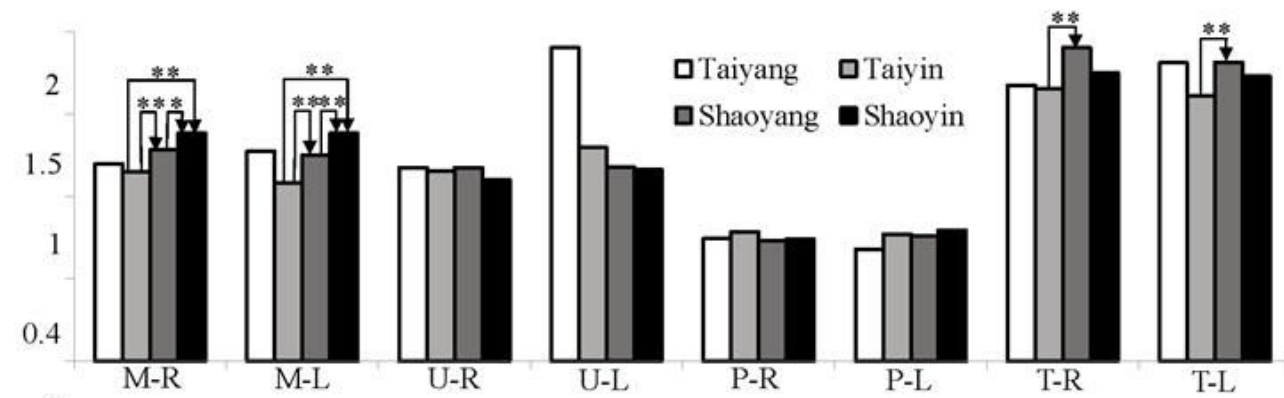

Note: ${ }^{*} P<0.05,{ }^{*} * P<0.01$. AMP. (amplitude of base to peek). One Way ANOVA.

Figure 6

Right and left side AMP. / DUR. ratio (average value) Note: ${ }^{*} \mathrm{P}<0.05,{ }^{*} \mathrm{P}<0.01$. AMP. (amplitude of base to peek). One Way ANOVA. 